\title{
Du mot à l'image, l'écriture scénaristique
}

\author{
Diane Henneton
}

Ordinairement, à la fin de chaque tournage, on retrouve les scénarios dans les poubelles du studio. Ils sont déchirés, salis, abandonnés. Très rares sont ceux qui en gardent un exemplaire, plus rares ceux qui les font relier, ou les collectionnent.

Jean-Claude Carrière ${ }^{1}$

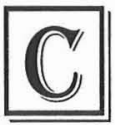

omme on allait autrefois retourner des masses de linge chez le fripier dans l'espoir d'y trouver un vêtement à sa convenance ou une étoffe, usée par endroits jusqu'à la trame, mais belle encore, pour se tailler un habit, c'est en fouillant les poubelles du cinéma que j'ai trouvé mon objet de travail. Parent pauvre des études cinématographiques et littéraires, le scénario constitue un objet de recherches encore largement inexploré.

Objet qui tend vers un autre objet, le scénario se tourne résolument vers l'avenir, vers l'ailleurs que sera le film. Il se projette vers l'acte à réaliser. Texte hybride, il est rarement pris comme objet d'étude, ce qui laisse d'autant plus perplexe que la question " littérature et cinéma » est abondamment débattue depuis les origines mêmes du septième art. Parmi les nombreux ouvrages qui traitent des interactions entre ces deux arts, peu abordent la question de l'écriture scénaristique, comme si elle était d'emblée exclue de cette problématique. Est-ce à dire que pour les auteurs de ces ouvrages critiques, elle n’a pas droit de cité au panthéon des œuvres littéraires? Ce n'est pas un de ses moindres paradoxes que d'être également

1 Exercice du scénario, FEMIS, 1990. 
souvent refoulée (dans le cadre universitaire) aux portes des études cinématographiques, comme la part impure, reniée de cet art composite.

Le défaut de littérarité de ces textes, d'ailleurs souvent pointé par les écrivains cinéastes qui souhaitent les écarter de la publication de leurs œuvres complètes, explique en partie leur disgrâce auprès des départements de littérature. A l'exception des dialogues pour lesquels les écrivains étaient souvent sollicités dans les années 40 et 50 , les préoccupations esthétiques sur la matière verbale sont en effet réduites, voire inexistantes dans ce que j'ai défini comme le scénario de tournage (par opposition au scénario postmontage remanié en vue d'une publication), le scénariste travaillant en prévision des matériaux constitutifs du film (image, son, mouvement, lumière, couleur, etc.) ce qui explique la déception du lecteur non averti face à cette écriture en souffrance. Quand l'objet littéraire se propose comme fin en soi, le script est un passage, un texte en devenir qui ambitionne de disparaître pour se projeter dans le film. Les professionnels du cinéma auxquels il est destiné n'en font eux-mêmes qu'une lecture chirurgicale en ne s'attachant la plupart du temps qu'aux lignes à partir desquelles ils vont devoir travailler. Ce mode de lecture est intimement lié à la nature même du script, qui est avant tout, non pas un texte donné à lire pour lui-même, mais un outil de travail.

Plus étonnant est à mon sens le silence dont on l'entoure dańs les départements de cinéma.

On pourra argumenter qu'il y a déjà bien assez à faire avec l'étude des films eux-mêmes, pourtant on crée bien des cours sur la distribution et la promotion des films ${ }^{2}$, alors que le scénario est tenu à l'écart ou qu'on l'aborde d'une manière tout à fait réductrice. Mais qu'appelle-t-on exactement scénario? On pourra s'étonner de cette question. Pourtant, s'il est un objet caméléon dans cet art neuf que constitue le cinéma, c'est bien le scénario, texte dont la nature changeante n’a guère été interrogée. A ce simple mot correspondent en effet bien des réalités. A la Bibliothèque du Film à Paris, par exemple, sous le terme générique de scénario, sont regroupés tous les documents de travail relatifs à l'élaboration des films (écriture, production, tournage, distribution). Dans une librairie, on pourra demander par exemple un scénario de Marguerite Duras, François Truffaut ou Jean Renoir. Or, les textes ainsi libellés par l'éditeur ou l'auteur lui-même sont en fait presque toujours des textes établis sur la base du film terminé ou bien des scénarios narrativisés après coup et qui s’apparentent plus ou moins à des romans. Il est extrêmement difficile d'ailleurs de

2 Cours d'ailleurs dont l'intérêt et la légitimité ne sont pas contestés ici. 
connaitre la véritable nature de ces textes qui sont publiés la plupart du temps sans précision aucune sur ce point.

L'incertitude qui entoure le texte même appelé scénario tient sûrement à l'étymologie du mot. Scénario désigne en effet deux réalités : il s'agit d'une part du canevas d'une œuvre, c'est-à-dire de l'histoire qu'elle raconte, d'autre part de l'outil de préparation d'un film présenté sous forme de texte.

La nature multiple des objets étiquetés « scénario " oblige, à mon sens, à un premier classement en trois grandes catégories.

1. Celle des documents relatifs à la production, au tournage et à la distribution qui n'ont rien, ou peu à voir avec le texte proprement dit du film.

2. L'ensemble de textes qui constituent comme la partition du film à réaliser, que leur rédaction soit antérieure ou contemporaine au tournage et que je désigne sous le terme de " scénario de tournage $»$.

3. La troisième catégorie que j'ai identifiée est celle du "scénario post-montage ", c'est-à-dire un texte établi en aval. Il peut s'agir d'un découpage technique établi sur la base du film, d'un " cinéroman », d'un « roman-cinéma », d'un « roman-cinéoptique », ou de " tout texte du film » remanié après coup. En dépit de tout l'intérêt qu'ils représentent, je ne les aborderai pas ici car tout l'enjeu de ma réflexion porte sur la seconde catégorie de textes.

Cette seconde catégorie recouvre cinq réalités qui correspondent aux différentes étapes de préparation du film : le synopsis, le traitement, le scénario également appelé script ou continuité dialoguée, le découpage technique - également appelé Bible, c'est dire l'importance qu'il revêt dans le processus de réalisation - et enfin le story board. A noter par ailleurs que dans les cas les plus fréquents où le cinéaste n'est pas l'auteur du scénario, une distinction est à établir entre "scénario de tournage " conçu par le scénariste et qui s'arrête au stade de la continuité dialoguée, et "scénario de tournage " conçu par le réalisateur sous forme de découpage technique et parfois de story board à partir du texte que lui a remis le scénariste. Dans le cas des écrivains-cinéastes, l'auteur est amené à effectuer le découpage technique et réalise même parfois un story board.

- Le synopsis se présente comme un exposé succinct du sujet (une à quatre pages environ) souvent destiné à convaincre les producteurs 
susceptibles de s'intéresser au projet. Il fournit avant tout du sens mais, comme le précisait Raymond Nouet dans son ouvrage sur la pratique scénaristique : «[...] c'est lui qui rend le moins compte de l'organisation structurelle du film³. »

- Le traitement (quelques dizaines de pages) consiste en l'élaboration de l'histoire en séquences. L'intrigue s'y développe et le scénariste établit en détail la structure dramatique du film (les transitions, l'évolution des personnages, etc. sont données dans ce document). Une esquisse de dialogue fait son apparition.

- La continuité dialoguée ou scénario est la dernière étape avant le découpage technique. Elle offre le découpage de l'histoire en scènes et séquences ainsi qu'une description du contenu de la bande sonore avec l'intégralité des dialogues ou des voix narratrices. Selon certains ouvrages, la réalisation d'une continuité exige de la part du scénariste une véritable connaissance des possibilités cinématographiques pour y adapter un sujet ou une œuvre purement littéraire, mais elle ne contient pas nécessairement des indications techniques. Dans la plupart des cas, c'est ce texte qui sera remis aux assistants, acteurs et techniciens, et qui servira de référence pour la mise en scène comme pour le montage des plans tournés.

- Le découpage technique est ordinairement rédigé par le réalisateur et il comporte toutes les informations techniques précises en vue du tournage (échelles de plans, mouvements d'appareil, effets sonores, effets spéciaux, etc.)

- Le story board apparaît parfois en fin de parcours. Il s'agit d'un découpage présenté comme une bande dessinée avec, en général, une vignette par plan.

La pratique de l'écriture scénaristique n'étant pas figée, les différents stades d'écriture présentés plus haut ne constituent en rien un parcours obligé dont il faudrait connaître toutes les étapes. Ainsi, certains textes sont à mi-chemin entre le synopsis et le traitement, des formes intermédiaires

3 Raymond Nouet, Comment écrire pour le cinéma, précis de technique du scénario, Agence littéraire du cinéma, 1957, p. 12. 
existent entre traitement et continuité dialoguée, ou entre scénario et découpage technique. Certains auteurs rédigent directement une continuité dialoguée, des cinéastes entament la réalisation directement à partir d'un traitement qu'ils ont rédigé eux-mêmes, créant ainsi des degrés variables d'improvisation allant du travail sur canevas, à l'écriture quotidienne sur le lieu et dans le temps mêmes du tournage.

Même ainsi définie, la " nature du scénario " reste encore le terrain de débats, voire même d'affrontements sur la question précise qui m'intéresse : celle de la mise-en scène. N'ayant pas trouvé en France de travaux universitaires sur ce sujet, je me suis alors penchée à la fois sur les scénarios eux-mêmes et sur les ouvrages destinés aux futurs scénaristes. Il n'est en effet pas indifférent de considérer dans le travail qui est le nôtre la nature même de l'enseignement dispensé dans les écoles qui forment les scénaristes. Les livres les plus intéressants pour mon étude sont publiés par la FEMIS et l'Ecole Louis Lumière. Ces ouvrages constituent de précieux documents sur la manière d'envisager l'écriture scénaristique (puisqu'ils traitent de son élaboration). Ils obéissent essentiellement à deux tendances : les uns s'attachent plutôt, voire exclusivement, au récit, les autres, sans négliger l'élaboration de l'histoire, insistent sur l'importance de l'écriture scénaristique comme travail incluant la mise en scène cinématographique. Parmi ces derniers, je citerai ceux de Jean Claude Carrière qui enseigne l'écriture scénaristique à la FEMIS ainsi que celui de Pierre Maillot, professeur à l'Ecole Louis Lumière. Dans L'Ecriture scénaristique, Pierre Maillot écrit : "Le scénario doit tout traiter : décors, lumières, jeu de l'acteur, costumes, et même, surtout, le style cinématographique ${ }^{4}$. " Il considère donc la mise en scène comme une partie constitutive du scénario et tout son ouvrage s'attache à développer cette dimension de l'écriture scénaristique chez ses lecteurs (qui sont aussi pour la plupart, étudiants dans des écoles de cinéma). Jean Claude Carrière qui partage la même vision du travail de scénariste note dans Exercice $d u$ scénario publié par la FEMIS : "Le scénario est la première forme d'un film. Il doit être imaginé, vu, entendu, composé et par conséquent écrit comme un film. Et le film même sera présent dans l'écrit, incrusté, précis, entrelacé, prêt à l'envol comme l'est le papillon, qui possède déjà tous ses organes et toutes ses couleurs sous une apparence de chenilles.»

4 Pierre Maillot, L'Ecriture cinématographique, Armand Colin, coll. Références, 1996, p. 230.

5 Exercice du scénario, FEMIS, 1990, p. 12. 
J'aurais beau jeu de ne présenter ici que le témoignage de scénaristes, c'est pourquoi j'ai également souhaité donner la parole à des cinéastes. Mais auparavant, il me semble nécessaire de poser deux jalons dans l'histoire du script. Le premier correspond à l'apparition du parlant qui a révolutionné l'écriture scénaristique : la nécessité de prendre en compte la bande sonore dans ses diversités (intégration de paroles mais aussi de bruits et de musique), et surtout le synchronisme de l'image et du son, imposèrent une prévision détaillée du montage, et donc une modification du script, tant dans sa forme, que dans son contenu. C'est dans cette période que le scénario prend conscience du rôle qu'il peut jouer dans la mise en scène et s'impose, si ce n'est comme une étape indispensable, du moins comme un passage souvent incontournable de la création cinématographique. Dans la plupart des scripts des années 40 et 50 , pour chaque plan, on trouve des indications non seulement de ce qui se passe, mais le cadre dessiné, le numéro de l'objectif à utiliser, etc. Cette mutation du scénario entraîne l'évolution du statut de scénariste qui devient alors la pièce maîtresse dans la constitution de l'équipe du film et le restera jusqu'à l'avènement de la Nouvelle Vague. Dans les années 40- 50, il arrivait que le nom d'un scénariste, comme celui d'Henry Jeanson par exemple, soit plus connu du public que celui du cinéaste. Il y avait là sans aucun doute un excès qui s'est retourné contre les scénaristes à l'avènement de $\mathrm{La}$ Nouvelle Vague. Dès 1948, l'article célèbre d'Astruc " Naissance d'une nouvelle avant-garde, la caméra-stylo ${ }^{6}$ " défend l'idée selon laquelle un réalisateur doit écrire lui-même ses scénarios.

Le second grand tournant dans l'histoire du script en France correspond à l'apparition de La Nouvelle Vague. Auparavant, un film tourné en studio pouvait se conformer très exactement au scénario et se présenter comme une interprétation fidèle du script. Mais dès qu'il fait le mur, pour utiliser une image peut-être un peu familière, il doit se colleter aux imprévus du monde extérieur, ce qui le contraint à assouplir sa forme. Cette nouvelle donne révolutionne l'écriture scénaristique qui doit alors s'adapter aux lieux de tournage. Mais c'est surtout le malaise qu'éprouvent les cinéastes tant vis-à-vis du scénariste que du scénario lui-même, et leur revendication d'être les auteurs complets du film, qui va influer sur le destin du script; le scénariste étant perçu comme un rival. Comme je l'ai expliqué plus haut, c'est dans le contexte de scénarios très directifs en matière de mise en scène que la Nouvelle Vague est apparue. Conscients de cette dimension de l'écriture scénaristique, les cinéastes de la Nouvelle

6 L'Ecran français, $\mathrm{n}^{\circ}$ 144, mars 1948. 
Vague vont justement s'en emparer. Il s'agit pour eux, à la fois de s'affranchir du rival que constitue le scénariste, et de renverser une conception de la création cinématographique qui ne voit dans le tournage que l'étape au cours de laquelle le réalisateur met au monde une œuvre dont la forme est déjà, pour une large part, arrêtée dans le script. Certains d'entre eux s'affranchiront de ce qu'ils voient comme un carcan et travailleront par improvisations. Figure emblématique de cette nouvelle manière d'aborder le cinéma, Godard explique : "Il y a deux grandes classes de cinéastes. Du côté d'Eisenstein et d'Hitchcock, il y a ceux qui écrivent leur film de façon la plus complète possible. Ils savent ce qu'ils veulent, ils ont tout dans la tête, ils mettent tout sur le papier. Le tournage n'est qu'une application pratique. Il faut construire quelque chose qui ressemble le plus possible à ce qui a été imaginé. Resnais est de ceux-là, et Demy. Les autres, du côté de Rouch, ne savent pas très bien ce qu'ils vont faire et ils cherchent. Le film est cette recherche ${ }^{7}$. "Je cite également Eric Rohmer qui écrit dans Six contes moraux $x^{8}$ : "L’ambition du cinéaste moderne, et qui fut aussi la mienne, est d'être l'auteur à part entière de son ouvre, en assumant la tâche traditionnellement dévolue au scénariste." "L'inquiétude que le scénariste fait planer sur la question de la paternité de l'œuvre est ici clairement exprimée par le cinéaste. Alors qu'il est l'auteur de ses propres scénarios, Rohmer va même plus loin en déclarant : "Je m'imaginais, dans ces films où la part du texte est primordiale, qu'en rédigeant celui-ci d'avance, $\mathrm{j}$ 'allais me priver, pour la durée du tournage, du plaisir de l'invention. Que ce texte fut de moi-même ou d'un autre, je répugnais à n'être que son servant [...] »9. Le rapport de vassalité qu'un cinéaste peut éprouver vis-à-vis du script est ici clairement énoncé.

A partir du moment où le réalisateur a pris le devant de la scène, et avec le retentissement que l'on sait, le scénariste est rentré dans l'ombre, mais n'en a, dans nombre de cas, pas moins poursuivi son travail d'écriture en prévision de la mise en scène, ce qui ne veut pas dire que le réalisateur ne travaillera pas contre ces indications par la suite. Depuis qu'ils ont fait l'objet d'une désaffection, rares sont les scénaristes qui ont l'occasion de s'exprimer publiquement. Ceux qui le font restent très discrets sur cette question. C'est le cas de Jean-Claude Carrière par exemple qui évite d'aborder la question des indications de mise en scène dans l'écriture

7 Jean-Luc Godard par Jean-Luc Godard, éd. Cahiers du cinéma, 1998, p. 2227

8 Roman, L'Herne, 1974, p. 10

9 p. 10-11. 
scénaristique quand il évoque un film dont il a écrit le scénario, alors que dans ses ouvrages destinés aux étudiants de la FEMIS, il revendique cette part de création. "Un scénario est déjà le film " écrit-il dans Exercice du scénario ${ }^{10}$. Peut-être faut-il nuancer cette affirmation en disant plutôt : " un scénario est déjà un film ", car on sait qu'au tournage, l'auteur même du script peut le changer complètement. Faut-il rappeler les propos de Truffaut qui disait tourner contre le scénario et monter contre le tournage? Ou l'exemple de Bresson qui, selon ses propres dires, écrivait ses scénarios avec une grande minutie, en indiquant par exemple les grosseurs de plan et le travail sur la bande-son puis changeait tout au moment du tournage? Encore faudrait-il vérifier, scénario de tournage en main, la véracité de ces déclarations.

Toujours sur cette question, les écrivains auteurs de scénarios constituent une catégorie tout à fait intéressante. En effet, habitués à être les maîtres de leur création, ils ne craignent pas de s'exprimer ouvertement sur la question de la paternité de l'œuvre, et certains d'entre eux revendiquent une part qui va bien au-delà de la seule écriture d'une fiction. Jean Cayrol, Alain Robbe-Grillet ou Marguerite Duras par exemple, expriment tous, et par la publication de leur script aussi, un désir de voir reconnaître leur apport à la mise en scène. Les films auxquels ces écrivains ont collaboré sont devenus des films de Resnais ${ }^{11}$, or cette unique signature n'apparaît pas toujours comme légitime à tous, et pas seulement aux auteurs de scénarios eux-mêmes. Par exemple, dans le Dictionnaire des films d'Henry Tulard, Alain Robbe-Grillet est ainsi présenté : "Ingénieur agronome, père du Nouveau Roman, il fit, comme scénariste, voler en éclats les structures traditionnelles du récit cinématographique, dans L'Année dernière à Marienbad dont il est, plus que Resnais, le véritable auteur $^{12}$. " Je cite à présent, une interview de Robbe-Grillet par Jean-Jacques Brochier :

J. J. B. : Est-ce que vous avez l'impression que votre travail de scénariste avec Resnais a été un travail de création?

A. R-G. : Oui, parce que ce n'était pas seulement un scénario. Ce que j'ai remis à Resnais [...] c'était déjà un film visualisé, c'est-à-dire que j'ai

10 Exercice du scénario, FEMIS, 1990, p. 2

11 Muriel, L'Année dernière à Marienbad, Hiroshima mon amour.

12 Dictionnaire du cinéma, Robert Laffont, 1992, p. 584. C'est nous qui soulignons. 
décrit à Resnais non pas une histoire, mais des images. Resnais a respecté de façon précise ces images, en inclinant cependant l'ensemble du côté de la psychologie.

J. J. B. : Pour un scénariste, décrire des images, c'est empiéter sur le rôle du metteur en scène.

R.-G : Absolument.

J. J. B. : Et le résultat vous a semblé fidèle à ce que vous aviez imaginé? R.-G. : C'était quand même un film de Resnais, et qui se plaçait dans la ligne de ses films. Mais quand je le voyais, je pouvais parfaitement y retrouver un film de moi. $»^{13}$

En guise de conclusion, rappelons que cette étude n'essaie en rien de nier la différence ontologique qui existe entre littérature et cinéma, entre le caractère sensible du spectacle filmé, fait d'images visuelles et de sons, et l'essence intellectuelle et abstraite de l'écriture, faite de mots et de phrases. Elle s'efforce seulement de rechercher une nouvelle approche du scénario compris comme lieu potentiel, mais pas obligatoire, d'un travail de mise en scène, dont l'implication et la précision varient d'un scénario à l'autre, d'une période de l'autre de l'histoire du cinéma.

13 " Deux activités parfaitement détachées l'une de l'autre ", Magazine littéraire, $\mathrm{n}^{\circ}$ 41, juin 1970, p. 15. 